\title{
Mendelian susceptibility to mycobacterial diseases due to complete IL12B deficiency
}

INSERM

\section{Source}

INSERM. (1999). Orphanet: an online rare disease and orphan drug data base. Mendelian susceptibility to mycobacterial diseases due to complete IL12B deficiency.

ORPHA:319558

Mendelian susceptibility to mycobacterial diseases (MSMD) due to complete interleukin12 subunit beta (IL12B) deficiency is a genetic variant of MSMD (see this term) characterized by mild bacillus Calmette-Guérin (BCG) infections and recurrent Salmonella infections. 